\title{
Correspondence
}

\section{How can a young person wait over 90 hours in an emergency department for a bed?}

We have recently been involved in a difficult case of a young person who remained in a local emergency department bed for over 90 hours while several specialty registrar (StR) doctors spent the majority of their on-call time attempting, and failing, to find an appropriate available bed. Over the course of this time, at least 40 units were contacted, numerous referral letters faxed and the case was handed over 6 times, all while the young person waited in an unsuitable setting that offered little to meet his mental health needs.

Although this is an extreme case, it reflects the trend we have observed of difficulty in finding beds, especially out of hours, and the fact that bed finding is becoming a major aspect of our on-call time. As Hillen \& Szaniecki demonstrated, ${ }^{1}$ the majority of referrals are made between the hours of $5 \mathrm{pm}$ and 9 am when daytime services are closed.

We have read with interest the findings of NHS England's Child and Adolescent Mental Health Services (CAMHS) tier 4 report $^{2}$ published in July 2014, which reported that the number of NHS-funded CAHMS tier 4 beds has increased by just 136 in the past 8 years, compared with a 284 rise between 1999 and 2006. This means that there were 1264 beds available nationally in January 2014. The BBC have declared this a problem of 'patchy provision' and, based on their findings, NHS England have identified a need and promised 'up to 50 new beds around the country with further beds moved according to need'. ${ }^{2}$ While this goes some way to providing more beds, this is only part of the problem.

There is currently no system to find out which beds are available at any given time and no external support to make the bed-finding process efficient. In our experience, weekly published lists are not representative of genuine availability and are quickly obsolete. There is no universal referral form so each referral necessitates new paperwork and often indiscriminate, convoluted processes of speaking to each individual bed manager, night nurses and support staff who do not have the responsibility to admit patients out of hours. A young person with complex problems and behaviours that need careful management is considered too risky for an open ward and so they are refused. Beds alone will not solve this; we need places that can be accessed out of hours with appropriate staffing levels and staff adequately trained to confidently manage the potential risk.

Because of these difficulties in admission, we are concerned that a prolonged wait in an emergency department will become more commonplace and that measures such as the Mental Health Act 1983 will be used to compel young people into admissions as their mental health deteriorates while waiting for a bed, and low-secure units become the default due to their ability to tolerate risk.

We are surprised that there is no centrally produced daily bulletin of national bed states and no provision to make NHS England accessible out of hours to assist with finding a bed. There is an urgent need for a better central system to ensure that vulnerable young people are not left in emergency departments without proper care.

1 Hillen T, Szaniecki E. Cyclic variations in demand for out-of-hours services in child and adolescent psychiatry: implications for service planning. Psychiatrist 2010; 34: 427-32.

2 CAMHS Tier 4 Report Steering Group. Child and Adolescent Mental Health Services (CAMHS) Tier 4 Report. NHS England, 2014 (http://www.england.nhs.uk/wp-content/uploads/2014/07/ camhs-tier-4-rep.pdf).

3 Buchanan M. Beds shortage for child mental health patients. BBC News Health 2014; 11 July. Available at: http://www.bbc.co.uk/news/ health-28255930 (accessed August 2014).

Gil Myers, Delphine Coyle, Christopher Kowalski and Ramya Srinivasan are all StRs in child and adolescent psychiatry working at the Tavistock and Portman NHS Foundation Trust, London, UK, email: gil.myers@nhs.net

doi: $10.1192 / p b .38 .5 .250$

\section{I prescribe, therefore I am?}

In their qualitative study, we imagine that Martean \& Evans ${ }^{1}$ captured the views of the majority of psychiatrists on their experiences of prescribing for personality disorder. Although we could identify with all of the themes determined as reasons for prescribing, we feel that the article highlighted a number of worrying trends within the profession that need to be addressed.

There appears to have been a shift away from a psychotherapeutic approach in psychiatry toward a distinct reliance on prescribing. The authors describe a theme of utilising prescribing as a method of communicating empathy. We would argue that it is disappointing if psychiatrists can only demonstrate empathy through the use of a prescription pad. It would seem that potential harm, in the form of possible serious side-effects, addiction, polypharmacy and indeed overdose facilitated by such a prescription may be more likely than benefit. Primum non nocere would suggest that, in the absence of convincing evidence for prescribing for personality disorders, the responsibility lies with the doctor to examine alternatives.

The authors themselves identify one potential solution in their recognition that 'problems as much or perhaps more than diagnosis may be crucial to explore for patients with personality disorder'. Problem-solving therapy has been shown to improve depression, hopelessness and personal problems in patients who self-harm ${ }^{2}$ and has demonstrated specific benefit as a preliminary measure for patients with a personality disorder. ${ }^{3}$ Perhaps this may be a useful initial intervention to avoid feeling helpless in such consultations. Longer-term options such as dialectical behaviour therapy and specialised counselling for trauma experienced in childhood allow deflection away from the prescription.

While we acknowledge that treating patients with personality disorders is often challenging, we believe the profession needs to move away from the notion of 'I prescribe, therefore I am'. Ultimately, the increased focus on psychotherapy in the updated curricula of both the Royal College of Psychiatrists and the College of Psychiatrists of 